\title{
RESPONSABILIDADE COMPARTILHADA E SUA INFLUÊNCIA NAS ATITUDES DE ESTUDANTES DE ADMINISTRAÇÃO DE UMA IES PRIVADA DE PORTO ALEGRE
}

\author{
SHARED RESPONSIBILITY AND ITS INFLUENCE ON \\ THE ATTITUDES OF MANAGEMENT STUDENTS AT \\ A PRIVATE UNIVERSITY IN PORTO ALEGRE
}

Recebido 19/08/2015• Aprovado em: 03/11/2015 Avaliado pelo sistema double blind review Editora Científica: Cláudia de Salles Stadtlober DOI: $10.13058 /$ raep.2016.v17n1.330

\section{CLÁUDIO DAMACENA damacena.claudio@gmail.com THAYS MARTINS DO NASCIMENTO PONTIFÍCIA UNIVERSIDADE CATÓLICA DO RIO GRANDE DO SUL}

\begin{abstract}
RESUMO
A perspectiva da coprodução em serviços vem ganhando espaço no contexto educacional. Caracteriza-se como um modelo educacional mais interacional e de responsabilidade compartilhada. Em outras palavras, os professores desenvolvem aulas que facilitam e motivam os alunos a produzirem as suas atividades, ou seja, coproduzem o aprendizado. Nesse contexto, a colaboração ativa, o diálogo, a interação, a mútua prestação de contas e a reflexão são aspectos intrínsecos da aprendizagem e podem ajudar a reduzir o impacto negativo de falhas que ocorrem nos serviços. Com o objetivo de explorar essa temática empiricamente, foi realizada uma pesquisa descritiva com 308 alunos de Administração de IES privada de Porto Alegre, com base em investigação semelhante produzida por Sierra (2009). Os resultados do estudo comprovam que a responsabilidade compartilhada é um componente crítico do aprendizado do aluno e sobre sua experiência de educação empresarial, pois o influencia, de forma determinante, em suas avaliações objetivas, subjetivas, cognitivas e emocionais.

Palavras-chave: responsabilidade compartilhada; experiência de ensino; coprodução em sala de aula.
\end{abstract}

\footnotetext{
ABSTRACT

Within education, co-production has been steadily gaining ground. It is characterized by an educational model that provides more interaction and shared responsibility. In other words, teachers develop lessons that motivate students to produce their activities and facilitate that process. In this context, active collaboration, dialogue, interaction, mutual responsibility and reflection are intrinsic aspects of learning and can help to reduce the negative impact of failures that occur in the services. In order to explore this theme empirically, descriptive research was conducted with 308 students from a management course at a private university, in Porto Alegre. The research was based on a similar investigation by Sierra (2009). The results show that shared responsibility is a crucial component in students' learning, as well as in their experience of business education, because of its determining influence over their objective, subjective, cognitive and emotional assessments.

Keywords: shared responsibility, teaching experience, co-production in the classroom.
} 


\section{INTRODUÇÃO}

Segundo Vargo e Lusch (2004), o consumidor está sempre envolvido na produção de valor, passando de "recurso operado" a "recurso operante". Nessa linha de pensamento, ainda que se fale em bens de consumo, a produção não se encerra no processo de manufatura, pois o consumidor precisa aprender a utilizar o produto e adaptá-lo as suas necessidades pessoais e situações de uso. Na prestação de serviços, o papel do cliente como coprodutor fica ainda mais evidente, pois o envolvimento entre as partes é maior.

Bitner et al. (1997) classificam os serviços de acordo com o nível de participação do cliente. Os serviços com nível de participação baixa correspondem àqueles nos quais o prestador efetua toda a produção e o cliente se comporta de forma receptiva, como, por exemplo, lavanderia e transportes aéreos. Em casos de participação moderada, o fornecedor presta o serviço mediante informações e orientações solicitadas pelo cliente, como no caso de serviços de contabilidade, cabeleireiro ou agência de propaganda. Por fim, em serviços com alto grau de participação, o cliente atua como cocriador ou coprodutor, casos nos quais pode contratar um serviço, pagar por ele, mas somente por meio de sua presença e participação ativa ele pode ser efetivamente concretizado. São exemplos de serviços desta categoria, personal trainers e serviços educacionais.

Tendo em vista a pesquisa que será abordada neste trabalho, vamos nos deter com mais atenção nestes últimos, os serviços educacionais, focalizando especificamente o nível superior de ensino e o curso de administração.

A perspectiva da coprodução em serviços vem ganhando espaço no contexto educacional. Caracteriza-se como um modelo educacional mais interacional e de responsabilidade compartilhada. Em outras palavras, os professores desenvolvem aulas que facilitam e motivam os alunos a produzirem as suas atividades, ou seja, coproduzem o aprendizado. Nesse contexto, a colaboração ativa, o diálogo, a interação, a mútua prestação de contas e a reflexão são aspectos intrínsecos da aprendizagem (SIERRA, 2009). Além disso, a responsabilidade compartilhada pode ajudar a reduzir 
o impacto negativo de falhas que ocorrem nos serviços (SIERRA; HEISER; MCQUITTY, 2009).

Alguns estudos sugerem que as trocas sociais que ocorrem no ambiente de serviços podem gerar emoções positivas ou negativas (LAWLER; THYE; YOON, 2000; SIERRA; HEISER; MCQUITTY, 2009; KU; HSU, 20I5), afetando, em algum grau, a experiência de serviço e a lealdade à marca.

As emoções resultantes de trocas sociais podem influenciar as relações sociais; ou seja, as emoções são redirecionadas para os indivíduos e as redes de indivíduos relacionados. Assim, os clientes que têm experiências positivas no local de um serviço podem desenvolver a lealdade com a mesma marca de serviço devido a uma experiência emocional positiva e consequente percepção de valor de troca. Da mesma forma, as emoções negativas geradas em um local de serviço podem afetar adversamente a lealdade para com essa marca de serviço. Pesquisas examinando essas relações são escassas (SIERRA; HEISER; MCQUITTY, 2009, p. II3).

Da mesma forma, as trocas sociais realizadas no ambiente acadêmico, mais especificamente em sala de aula, além de afetarem o aprendizado, impactam, também, na experiência do serviço e na lealdade à marca da instituição de ensino. Assim, a criação de um ambiente de ensino interativo deve motivar estudantes a participar ativamente das aulas, melhorando o seu desenvolvimento de habilidades (PALADINO, 2008; KARNS, 2006; YOUNG, 2005). as aulas devem ser ministradas com metas claras, com professores entusiasmados, que proporcionem alta interação e feedback de apoio. (Young, 2005). A motivação intrínseca dos estudantes pode ser mais estimulada, definindo expectativas claras e razoáveis de coprodução de valor.

Atentando para a relevância da percepção de responsabilidade compartilhada por parte dos alunos, Sierra (2009) conduziu uma pesquisa, avaliando os efeitos da percepção de responsabilidade compartilhada sobre alguns fatores: (I) a imagem dos alunos em relação à experiência de aprendizagem; (2) as emoções dos alunos em relação à experiência; (3) suas atitudes; e (4) suas intenções de cursar mais disciplinas com o professor. Sierra (2009) testou as hipóteses em uma amostra coletada nos Estados Unidos e descobriu que as percepções de responsabilidade compartilhada 
para a aprendizagem do aluno estão positivamente relacionadas às respostas comportamentais, emocionais e nas atitudes dos alunos em relação à experiência de educação em marketing. E também que essa responsabilidade compartilhada se relaciona positivamente com as notas dos alunos.

Os resultados da pesquisa são válidos para estudantes de Administração americanos. No caso de estudantes brasileiros, os resultados da pesquisa seriam os mesmos? Buscando a resposta para essa pergunta, foi realizado um estudo com estudantes brasileiros de Administração de uma universidade privada brasileira. É sobre esse estudo que trata o presente trabalho. Para abordá-lo, tendo em vista o contexto apresentado, o referencial teórico é exposto no item a seguir, sendo sucedido pelo item em que consta o método e pelo item de resultados. Finalmente, são apresentadas as conclusões do estudo, bem como suas referências.

\section{COPRODUÇÃO E RESPONSABILIDADE COMPARTILHADA}

A responsabilidade compartilhada pela produção ou coprodução de valor é imprescindível em serviços que exigem grande participação do cliente (BITNER et al., 1997). Serviços educacionais, entre outros, representam muito bem essa necessidade participativa dos clientes. Convém ressaltar, portanto, o que significa valor nesse contexto. O significado de valor pode ser bastante diferente para os públicos que interagem nos serviços educacionais. Brambilla e Damacena (2012) afirmam, por exemplo, que instituição de ensino e alunos devem se preocupar com o resultado efetivo do serviço, ou seja, que as competências e habilidades previstas tenham sido desenvolvidas e comprovadas. Afirmam, ainda, os autores:

Por um lado, os estudantes estão preocupados em aprender (ou desenvolver as competências necessárias para o mercado de trabalho), não sendo reprovados. Assim, a experiência desse processo de cocriação será satisfatória. Pelo lado do professor, o objetivo é que os alunos desenvolvam as competências e que o avaliem de forma positiva. Para a instituição, é importante que os estudantes tenham desenvolvido as competências previstas, o que poderá ser provado em testes governamentais, e que o aluno não abandone a instituição e, ainda, possa ser um indicador de potenciais estudantes (na prospecção de alunos). 
Toda a sociedade necessita de profissionais hábeis à resolução das tarefas cotidianas, e, no caso dos negócios, exemplos como condução de empresas e organizações deixam evidente a importância dos melhores resultados cocriados na preparação destes futuros trabalhadores (BRAMBILLA; DAMACENA, 20I2, p. 456). A coprodução ou responsabilidade compartilhada entre aluno e professor está ligada positivamente aos resultados desejáveis da aprendizagem, como o pensamento crítico, as notas e a sincronia do desempenho ao longo da carreira após a graduação (CARINI; KUH; KLEIN, 2006). A Responsabilidade Compartilhada é a dependência mútua ou responsabilidade para o sucesso de um resultado de serviço (SIERRA, 2009; SIERRA; HEISER; MCQUITTY, 2009).

Obviamente, um ensino superior eficaz envolve a mistura adequada de fatores físicos, infraestrutura da universidade e arranjo da sala de aula, com fatores relacionados com o desempenho dos professores, como entusiasmo, experiência e estilo de ensino (HILL; LOMAS; MACGREGOR, 2003). Há outros fatores importantes, entretanto, como é caso da percepção dos alunos acerca de sua responsabilidade no processo de aprendizagem.

$\mathrm{Na}$ Teoria da Troca Social de Lawler (200I), relaciona-se o sucesso de uma troca social com o grau de responsabilidade compartilhada de cada parte envolvida: os participantes de uma troca social, que partilham a responsabilidade, também compartilham os resultados emocionais. Relações sociais que se originam por meio de responsabilidade mútua para a criação de resultados bem-sucedidos de intercâmbio podem desencadear respostas emocionais positivas dos clientes de serviço, e o relacionamento afetivo gera uma fidelização do cliente (SIERRA, 2009; GUSTAFSSON; JOHNSON; ROOS, 2005).

Os professores são responsáveis pela aprendizagem dos alunos, pela preparação das aulas, discussões de líderes de classes e atividades desafiadoras com tarefas de aprendizagem, incentivando, assim, a coprodução dentro da sala de aula, como facilitadores, dando mais responsabilidade aos alunos (NIEMI, 2002) e colocando-os como membros ativos da sua aprendizagem, incentivando: o preparo do aluno para a aula, sua participação nas discussões em classe e seu esforço no desenvolvimento das tarefas solicitadas (SIERRA, 2009).

A aprendizagem ocorre por meio dessa mediação de interação social. $O$ conhecimento não é de posse individual; deve ser socialmente partilhado e 
emerge com a participação dos alunos em atividades socioculturais. Novos métodos de ensino consistem em uma aprendizagem mais independente, arranjos mais colaborativos, tarefas e projetos mais abertos, permitindo aos estudantes colaborarem uns com os outros (NIEMI, 2002).

Todo esse trabalho dos professores, de envolver seus alunos e colocar em prática uma maior motivação no ensino, é parte integrante de como instituições de ensino devem desenvolver e definir sua visão, diretrizes e seus objetivos educacionais (BAYLEI, 2000).

\section{A responsabilidade compartilhada e a Instituição de Ensino}

Para estimular os alunos a coproduzirem, os professores precisam aprender os fatores que os estimulam e a forma certa para isso, para que os estudantes criem expectativas positivas em relação a sua participação. Seus comportamentos de coprodução são de diferentes formas: dentro e fora da sala de aula, em atividades individuais ou em grupo. (KOTZE; PLESSIS, 2003). Além da capacitação dos professores, quando uma instituição se propõe a atrair o aluno, ela precisa criar uma experiência educacional capaz de satisfazer as necessidades desse aluno (NG; FORBES, 2009). Indo além do ensino convencional, pois, se o resultado de um sistema de educação em negócios é preparar membros de sua comunidade para o mercado, então é necessário incentivar o pensamento crítico entre os seus alunos (GIBBS, 200I).

$\mathrm{Na}$ relação de ensino e aprendizagem entre professor e aluno são enfrentados alguns desafios, e somente haverá resultados positivos se os dois trabalharem juntos (CHUNG; MCLARNEY, 2000). Não há serviço bemsucedido sem a participação de ambas as partes: no caso de aluno e professor, ambos têm que perceber que há valor agregado no serviço do ensino. Um passo importante para a criação de valor em sala de aula é o professor entender a importância da participação do aluno (CURRAN; ROSEN, 2006; CHUNG; MCLARNEY, 2000; HALBESLEBEN; BECKER; BUCKLEY, 2003) com um entendimento de que o profissional (professor) e o cliente (aluno) desenvolvem um relacionamento ao longo do tempo e, isso, inerentemente envolve interação. Para o aluno conseguir os resultados de aprendizagem esperados, deve empenhar um mínimo de esforço em sala de aula, sendo 
ele um cliente em um ambiente educacional, o ideal é ter um professor que o desafie, guie, e o engaje na sua busca por conhecimento (BAILEY, 2000) e por uma experiência de ensino positiva. Esses objetivos educacionais mostram que os alunos têm propensão a serem desafiados a expandir o seu potencial. Usando-se um quadro aluno-como-cliente, fornece-se tanto ao professor quanto ao aluno uma perspectiva adequada para a construção de um ambiente de aprendizagem bem-sucedida (BAILEY, 2000).

A intenção de programas de ensino mais interativos não é apenas entregar o conhecimento, mas também aumentar a confiança do aluno, buscando soluções criativas baseadas nos resultados dessa aprendizagem, sendo relevante para a avaliação de sucesso da disciplina e do curso (DUKE, 2002). Os ambientes de sala de aula tradicionais são ainda a maioria, e a criação de ambientes de aprendizagem em que o aluno se envolva ativamente com o professor tem o potencial de estimular o desenvolvimento da aprendizagem, tanto experimentalmente como cognitivamente (YOUNG, 2005; HALBESLEBEN; BECKER; BUCKLeY, 2003). Young (2005) afirma, ainda, que práticas de sala de aula em que se desenvolve uma aprendizagem ativa do mundo real, seguindo objetivos claros e enfatizando o desempenho individual, produzem estudantes com maior autonomia e com melhor desempenho nas avaliações.

Sendo analisada essa interação professor-aluno como uma troca onde cada um tem o seu papel e a sua responsabilidade, as instituições devem estar preparadas para utilizar essas relações em seu benefício. A excelência no ensino é agora um imperativo competitivo na maioria das escolas de negócios (AUSTER; WYLIE, 2006). Os resultados adquiridos devem ser avaliados pelas Instituições de ensino podendo ajudá-las a tomar decisões estratégicas e aumentar sua vantagem competitiva, além de manter seu método de ensino mais dinâmico e desafiador ao aluno. A Instituição de ensino pode fornecer um ambiente respeitável para o público, indo além dos objetivos lucrativos, colocando todos os participantes dessa troca a ouvirem e se adaptarem, com um sistema de ensino superior em que se possa, de fato, confiar (GIBBS, 200I). O valor da empresa terá de ser criado conjuntamente por ela - Instituição/Professor - e pelo consumidor - aluno - (PRAHALAD; RAMASWAMY, 2004). 
Os efeitos da responsabilidade compartilhada são semelhantes aos descritos pela chamada aprendizagem ativa, que pode ser definida como qualquer trabalho que influencia os alunos a fazerem as atividades e a pensarem sobre elas (BONWELL; EISON, I99I). E, de acordo com Johnson, Johnson e Smith (199I), a aprendizagem ativa é o uso instrucional de pequenos grupos de alunos para que trabalhem em conjunto para maximizar a sua própria aprendizagem e auxiliar no desenvolvimento de cada um.

Essa interação entre os professores e alunos, em uma comunidade de aprendizagem, está positivamente associada ao engajamento do aluno, seu desempenho e seu envolvimento em atividades extracurriculares, juntamente com a sua integração acadêmica, ganhos associados com o comparecimento às aulas e, em geral, satisfação com a experiência da faculdade (ZHAO; KUH, 2004; KAENDLER et al., 2015).

As instituições de ensino que mantêm essa metodologia de aprendizagem impulsionam o envolvimento dos alunos, o que, por sua vez, leva a uma série de resultados educacionais positivos, como a imagem de alta qualidade (PIKE; KUH; MCCORMICK, 2OII; MOSTAFA, 20I5).

As instituições de ensino vêm se reformando e se adaptando a essas mudanças, que trazem resultados positivos no que se refere à visão dos alunos em relação a elas. O movimento para criar tabelas de classificação globais para as universidades é simbolicamente o indicador mais poderoso de que os valores de mercado foram incorporados no setor universitário (LYNCH, 2006). Para ter uma boa percepção diante dos seus alunos, as instituições devem buscar essa coprodução.

\section{Efeitos da responsabilidade compartilhada}

A inseparabilidade das partes na troca de serviços está positivamente relacionada com a percepção de responsabilidade compartilhada, e com as emoções associadas a essa interação. Ou seja, a responsabilidade compartilhada pode gerar emoções positivas (SIERRA; MCQUITTY, 2005; SIERRA; HEISER; MCQUITTY, 2009). A percepção de responsabilidade compartilhada na troca de serviços leva os consumidores a experimentarem emoções positivas nesse ambiente de interação, afetando a sua vontade de adquirir o serviço novamente (SIERRA; MCQUITTY, 2005; SIERRA, 2009). 
Essas trocas sociais produtivas geram relações favoráveis entre os participantes e criam recompensas na forma de resultados atitudinais, emocionais e comportamentais positivos, que podem ser compartilhados por todos os envolvidos na troca (LAWLER, 200I).

Em outras palavras, a relação de troca social é uma variável mediadora em que participam operações vantajosas e equitativas entre relações fortes, e esses relacionamentos podem produzir um comportamento de trabalho eficaz e atitudes positivas (CROPANZANO; MITCHELL, 2005). A parte que cabe aos professores é manter essa relação ativa, explorando as atitudes positivas dos alunos, para mostrar de que maneira estes podem alcançar os resultados almejados no curso.

Cada aluno tem um desempenho e uma capacidade de aprendizagem, e a clareza do papel dos estudantes ajuda no seu desenvolvimento e está positivamente relacionada com a sua participação em atividades de ensino e na sua aprendizagem (SIERRA; HEISER; MCQUITTY, 2009; KOTZE; PLESSIS, 2003). Por isso, as experiências de aprendizagem dos alunos podem ser melhoradas quando eles compartilham a responsabilidade pela aprendizagem de sucesso com professores (SIERRA, 2009).

A Teoria da Troca Social (LAWLER, 200I) sugere que relações formadas em uma troca social, nesse caso, a relação entre professor e aluno, são fontes de emoções para as partes dependentes do nível de responsabilidade compartilhada em uma troca bem-sucedida.

A troca social compreende ações contingenciais nas reações gratificantes dos outros, o que, ao longo do tempo, pode prever mutuamente transações gratificantes e relacionamentos (CROPANZANO; MITCHELL, 2005).

Além disso, o ensino recíproco, em que professores e alunos se envolvem na aprendizagem por meio do diálogo, leva a ganhos favoráveis aos estudantes. A coprodução e a prática interativa entre professor-aluno para atingir os objetivos levam a uma maior satisfação dos alunos sobre a sua experiência de aprendizagem.

A Teoria Relacional de Coesão (LAWLeR; THYE; YOON, 2000) propõe que as emoções resultantes de uma troca social podem ser atribuídas a uma relação em que cada participante contribui para um resultado benéfico. 
Participantes que auxiliam para as trocas sociais produtivas apresentam resultados emocionais mais positivos e fortes do que os participantes que não cooperam para a troca. Para os estudantes, a interação professor-aluno pode levar ao aumento da motivação intrínseca para a sua aprendizagem (YOUNG, 2005). Ao coproduzir a experiência de aprendizagem, estudantes desempenham dois papéis importantes na criação de um serviço, ou seja, o resultado como um recurso produtivo e como um contribuinte para qualidade, satisfação e valor. Como recurso produtivo, os alunos trazem consigo o seu intelecto, linguagem e habilidades de comunicação (NG; FORBES, 2009).

A percepção da sua capacidade e de suas atribuições de participação no curso tem sido usada como um fator de sucesso que aumenta a responsabilidade do estudante sobre o seu próprio comportamento de aprendizagem. A sua motivação em participar das atividades de ensino também está relacionada positivamente com a sua aprendizagem (YOUNG, 2005; KOTZE; PLESSIS, 2003).

A responsabilidade compartilhada salienta elementos sociais de aprendizagem. Por exemplo, na capacidade colaborativa em resolução de problemas, os alunos partilham conhecimento como ferramentas para alcançar processos mais profundos de aprendizagem e, em muitos casos, para também conseguir melhores resultados/notas. Isso é: a sua participação em discussões, diálogos e reflexões compartilhadas mutuamente resulta em uma melhor aprendizagem do aluno. Por esses motivos, tem atraído fortes defensores nas instituições que procuram alternativas aos métodos tradicionais de ensino (PRINCE, 2004), podendo ser uma maneira de o aluno se engajar e tomar atitudes ativas nas atividades da sala de aula (PRINCE, 2004; AUSTER; WYLIE, 2006), desenvolvendo o aprendizado colaborativo.

Um estudante que tem uma experiência emocional positiva com um professor durante o semestre pode procurar aulas adicionais com esse professor em um semestre posterior, ao passo que as emoções negativas podem afetar a disposição do aluno em seguir tendo aulas com um mesmo professor (LAWLER; THYE; YOON, 2000; SIERRA, 2009).

Estando o aluno motivado, podendo ver que a interação é positiva e produz emoções igualmente positivas e atitudes em relação ao aprendizado, 
a aula se torna mais agradável e atraente a ele, tornando os professores que utilizam dessa coprodução em sala de aula mais procurados pelos alunos, o que resulta em um retorno as suas outras disciplinas.

Dessa forma, surgem, como hipóteses de pesquisa, as que seguem:

Hr: Quanto maior a percepção do estudante de que ele compartilha a responsabilidade dos resultados de aprendizagem da disciplina, mais favorável será a imagem que ele formará em relação à experiência de aprendizagem na disciplina.

H2: Quanto maior a percepção do estudante de que ele compartilha a responsabilidade dos resultados de aprendizagem da disciplina, mais positivas serão as emoções dele em relação à experiência de aprendizagem na disciplina.

H3: Quanto maior a percepção do estudante de que ele compartilha a responsabilidade dos resultados de aprendizagem da disciplina, mais favorável será a atitude que ele formará em relação à experiência de aprendizagem na disciplina.

H4: Quanto maior a percepção do estudante de que ele compartilha a responsabilidade dos resultados de aprendizagem da disciplina, maior será sua intenção de cursar mais disciplinas com o professor. 


\section{MÉTODO}

O método utilizado foi o descritivo-quantitativo, que tem como característica básica a busca pela solução de problemas por meio de coleta de dados por questionários estruturados aplicados à amostra considerável (MALHOTRA, 20I2). A coleta de dados foi realizada durante o mês de outubro de $2014 \mathrm{em}$ uma universidade particular do Rio Grande do Sul, Brasil. A amostra final é composta de 308 estudantes, sendo que $52 \%$ são do sexo masculino e têm, em média, 23 anos, e $87 \%$ residem em Porto Alegre. Nas subseções que seguem, são descritos (I) o desenvolvimento do instrumento de coleta de dados e (2) o método de análise utilizado.

O instrumento original de coleta (vide Anexo A) foi adaptado com 27 variáveis, medindo a responsabilidade compartilhada (RC; 5 itens), resposta emocional em direção à experiência de aprendizado (EMOC; 6 itens), resposta atitudinal em direção à experiência em aprendizado (ATT; 4 itens), intenções de ter outra aula com o mesmo professor (INT; 5 itens), e imagem percebida da experiência em aprendizado (IMG; 7 itens). Foi utilizada uma escala Likert, da mesma forma que Serra (2009), sendo que o ponto " $\mathrm{I}$ " expressava "discordo totalmente", e o ponto " 7 " expressava "concordo totalmente".

O instrumento de coleta foi devidamente traduzido por três tradutores: uma tradução do inglês para o português feita pelo tradutor I; do português para o inglês, pelo tradutor 2; e do inglês novamente para o português, pelo tradutor 3. Concluído o processo de tradução reversa, a etapa seguinte foi à adaptação para o contexto da pesquisa. Foi submetido a dois professores especialistas (um, coordenador do curso de Administração da Universidade onde foram aplicados os questionários, e, outro, professor da graduação e da pós-graduação strictu-sensu em Administração da mesma Universidade) para que avaliassem a tradução e fizessem sugestões. Com base nas alterações sugeridas, obteve-se a versão final do instrumento de coleta de dados.

Foi realizado um pré-teste com ro alunos do curso de administração, que também fizeram suas sugestões sobre a aplicabilidade do instrumento. Os respondentes foram orientados a utilizar, como referência, para responder aos itens, a disciplina que estavam cursando e tendo o melhor desempenho 
no aspecto acadêmico e na relação com o professor.

Foi realizada uma análise fatorial exploratória e uma análise fatorial confirmatória. O método de extração utilizado foi o varimax, por estabelecer uma separação mais clara dos fatores, e a validade de confiabilidade das escalas foi realizada por meio do Alfa Cronbach. Os dados também foram analisados por meio de equações estruturais com o auxílio do software SmartPLS 3 . 


\section{RESULTADOS}

\section{ANÁLISE FATORIAL EXPLORATÓRIA}

Os testes Kaiser-Meyer-Olkin (KMO) verificam o grau de influência ou o ajuste dos dados à análise fatorial (Tabela I). A análise mostra que o teste Kaiser-Meyer-Olkin (KMO), que mede a adequação da amostra, foi de ,94I; ou seja, os dados são adequados para a análise fatorial exploratória. No teste de Barlett's, $\chi: 7847,005, \mathrm{p}<0,000$, os fatores extraídos explicam $76 \%$ da variância total. Foi utilizado o Alfa de Cronbach para avaliar a confiabilidade interna de cada uma das variáveis latentes pesquisadas. Todos os Alfas foram maiores que 0,7 , mostrando que as escalas utilizadas podem ser consideradas confiáveis (Tabela I).

Tabela I Resultados das cargas e Alfa de Cronbach

\begin{tabular}{|llllll|}
\hline Construto & $\begin{array}{l}\text { Variância } \\
\text { explicada (\%) }\end{array}$ & $\begin{array}{l}\text { Carga dos fatores } \\
\text { (mínimo e máximo) }\end{array}$ & $\begin{array}{l}\text { Número } \\
\text { de itens }\end{array}$ & $\begin{array}{l}\text { Número } \\
\text { de itens } \\
\text { excluídos }\end{array}$ & $\begin{array}{l}\text { Alfa de } \\
\text { Cronbach }\end{array}$ \\
\hline $\begin{array}{l}\text { Responsabilidade } \\
\text { Compartilhada }\end{array}$ & 58,30 & 0,75 a 0,80 & 5 & 0 & 0,821 \\
\hline Resposta Emocional & 74,32 & 0,80 a 0,91 & 6 & 0 & 0,930 \\
\hline Resposta Atitudinal & 78,91 & 0,86 a 0,91 & 4 & 0 & 0,911 \\
\hline Intenções & 88,19 & 0,91 a 0,95 & 5 & 0 & 0,967 \\
\hline Imagem & 78,74 & 0,85 a 0,91 & 6 & 0 & 0,955 \\
\hline
\end{tabular}

\section{VALIDADE CONVERGENTE E DISCRIMINANTE}

Os dados apresentados nas Tabelas 2 e 3 mostram que os construtos apresentam confiabilidade composta, validade convergente e discriminante. As cargas de todos os itens ultrapassaram 0,7 e todos os índices de confiabilidade composta (CC) foram superiores a 0,87 , atestando, assim, a validade convergente dos construtos (HAIR; RINGLE; SARSTEDT, 2OII; FORNELL; LARCKER, I98I). As variâncias médias extraídas (no inglês, Average Variance Extracted - AVE) foram todas superioriores a 0,58. Comparando a raíz 
quadrada das Ave com as correlações, pode-se verificar que os requisitos da validade discrimante são atendidos (FORNELL; LARCKER, I98I). Ou seja, as variâncias médias extraídas são superiores a o,5 e sua raiz quadrada é superior às correlações.

Tabela 2 Estatísticas descritivas e de confiabilidade e validade das variáveis

Itens da Escala $\quad$ Cargas

Responsabilidade Compartilhada (média $=5,17$ desvio padrão $=1,08 \mathrm{CC}=0,875 \mathrm{AVE}=$ 0,584)

- Devido ao importante papel que eu e o professor desempenhamos na minha educação,

0,750 eu sinto como se tivéssemos trabalhado juntos, como iguais, para fazer da minha experiência de aprendizado um sucesso.

- Eu percebo certo nível de responsabilidade tanto da minha parte quanto da do professor $\quad 0,759$ para fazer com que minha experiência de aprendizado seja bem-sucedida.

- O professor e eu contamos um com o outro para fazer dessa experiência de aprendizado $\quad 0,814$ um sucesso.

- O professor e eu somos, ambos, responsáveis pelo êxito educacional desta disciplina.

- O professor e eu estamos, ambos, em controle dos resultados educacionais desta

0,792 disciplina.

\begin{tabular}{|c|c|}
\hline - Estou feliz com a formação em Administração que eu estou recebendo. & 0,853 \\
\hline - A formação em Administração que eu estou recebendo é encantadora. & 0,802 \\
\hline - Estou satisfeito(a) com a formação em Administração que estou recebendo. & 0,913 \\
\hline - Estou contente com a formação em Administração que estou recebendo. & 0,897 \\
\hline - A experiência que estou tendo em educação em Administração é agradável. & 0,809 \\
\hline - É gratificante a formação em Administração que estou recebendo. & 0,894 \\
\hline
\end{tabular}

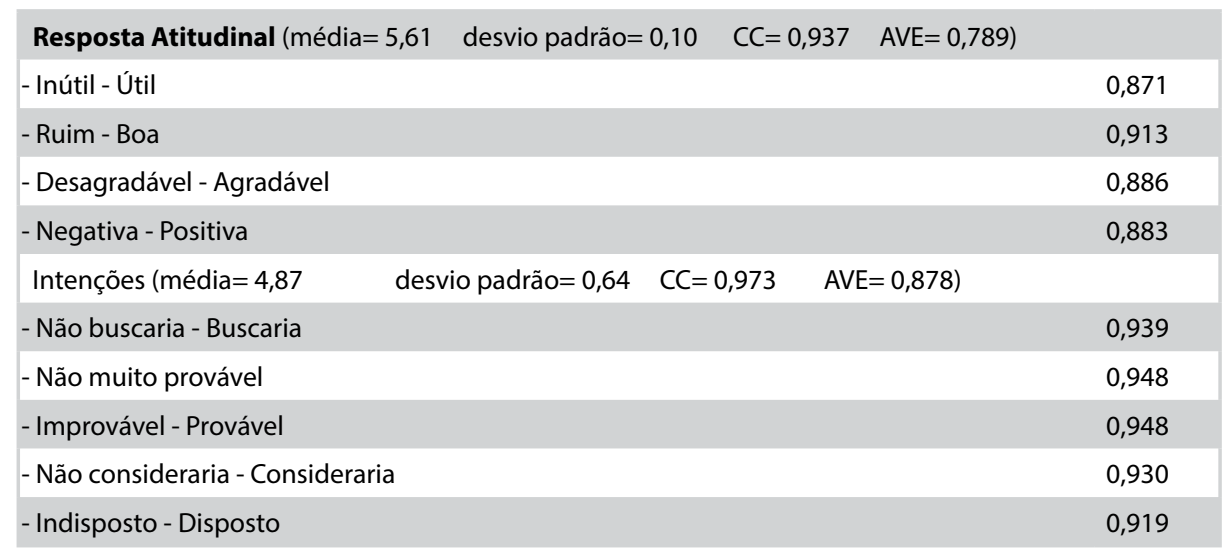




\begin{tabular}{ll} 
Imagem (média= 5,55 $\quad$ desvio padrão=0,07 CC=0,963 AVE=0,788) & \\
\hline - Não tem credibilidade - Tem credibilidade & 0,867 \\
\hline - Não prestigiosa - Prestigiosa & 0,909 \\
\hline - Não é respeitável - É respeitável & 0,910 \\
\hline - Irresponsável - Responsável & 0,891 \\
\hline - Não honesto - Honesto & 0,849 \\
- Baixa qualidade - Alta qualidade & 0,876 \\
\hline - Não confiável - Confiável & 0,907
\end{tabular}

Tabela 3 Fornell-Larcker Criterium - validade discriminante

\begin{tabular}{|c|c|c|c|c|c|}
\hline & ATT & EMOC & IMG & INT & $\mathbf{R C}$ \\
\hline ATT & 0,8883 & & & & \\
\hline EMOC & 0,6664 & 0,8625 & & & \\
\hline IMG & 0,7102 & 0,6291 & 0,8874 & & \\
\hline INT & 0,5531 & 0,4080 & 0,5526 & 0,9369 & \\
\hline $\mathbf{R C}$ & 0,5213 & 0,6241 & 0,5030 & 0,2769 & 0,7639 \\
\hline
\end{tabular}

\section{TESTE DAS HIPÓTESES}

Os resultados finais da análise, apresentados na Tabela 4, dão suporte a todas as hipóteses, visto que as cargas das relações possuem significância. Quanto maior a percepção do estudante de que ele compartilha a responsabilidade dos resultados de aprendizagem da disciplina, mais favorável é a imagem que ele forma em relação à experiência de aprendizagem na disciplina $(\beta=0,503$, $t=\mathrm{IO}, 8$ e poder explicativo igual a $25 \%$ ), mais positivas são as emoções dele em relação à experiência de aprendizagem na disciplina $(\beta=0,624, t=16,9 \mathrm{e}$ poder explicativo igual a $39 \%$ ), mais favorável é a atitude que ele forma em relação à experiência de aprendizagem na disciplina $(\beta=0,52 \mathrm{I}, t=\mathrm{I} 2,6 \mathrm{e}$ poder explicativo igual a $27 \%$ ) e maior é sua intenção de cursar mais disciplinas com o professor $(\beta=0,277, t=5,4$ e poder explicativo igual a $7,7 \%)$. 
Tabela 4 Resultados dos testes das hipóteses

\begin{tabular}{|lllll|}
\hline Relação testada & $\boldsymbol{\beta}$ & $\mathbf{t}$ & Sig. & $\mathbf{R}^{\mathbf{2}}$ \\
\hline Responsabilidade Compartilhada -> Resposta Atitudinal & 0,521 & 12,650 & 0,000 & 0,272 \\
\hline Responsabilidade Compartilhada -> Resposta Emocional & 0,624 & 16,984 & 0,000 & 0,390 \\
\hline Responsabilidade Compartilhada -> Imagem & 0,503 & 10,870 & 0,000 & 0,253 \\
\hline Responsabilidade Compartilhada -> Intenções & 0,277 & 5,446 & 0,000 & 0,077
\end{tabular}




\section{CONCLUSÕES E IMPLICAÇÕES}

O presente estudo apresenta contribuições vinculadas às demandas indicadas na área do marketing de serviço, (I) analisando a interação do professor e aluno em sala de aula, onde a coprodução pode ser observada (CHUNG; MCLARNEY, 2000), (2) no que se refere à responsabilidade compartilhada $\mathrm{e}$ sua reflexão na experiência de aprendizagem do aluno (SIERRA, 2009, 2005; LAWLER; THYE; YOON, 2000; LAWLER, 200I).

Assim como no estudo de Sierra (2009), foram encontradas relações positivas e significativas em todas as hipóteses, verificando assim que a aplicação dessa escala em um novo contexto teve uma boa adaptação, e o fato de terem se confirmado (as hipóteses) reforça a robustez da escala e suas adequações realizadas para a aplicação deste estudo. As percepções dos estudantes de RC para a sua experiência em aprendizado foram positivas e houve um nível elevado de propensão de alunos a interagirem gerando a coprodução em sala de aula.

Estudos realizados ressaltando a importância da responsabilidade compartilhada nas trocas sociais (SIERRA; MCQUITTY, 2005; SIERRA, 2009; LAWLER; THYE; YOON, 2000; LAWLER, 200I), e o presente trabalho vêm oferecer uma visão sobre a afirmação da sua importância em outros contextos, podendo, assim, garantir, para as instituições de ensino brasileiras, uma melhor compreensão sobre a relação entre professor e aluno em sala de aula.

Avaliando todos os construtos e seus resultados estatísticos, o item que obteve a maior carga foi Resposta Emocional, com 0,624, e $\mathrm{r}^{2}$ : 0,39. Então, $39 \%$ das relações emocionais foram explicadas pela responsabilidade compartilhada, que é um construto que avalia o grau de felicidade com a formação que o aluno está tendo, se ele está satisfeito e se é gratificante. Sierra (2009, p. 2), nessa mesma linha de raciocínio, afirma:

As emoções positivas geradas no intercâmbio social podem tornar-se fontes de valor para os participantes. As emoções resultantes de trocas sociais têm a capacidade de influenciar as relações sociais, onde as emoções são redirecionadas para as pessoas e as redes de pessoas relacionadas, em última análise, influenciar respostas comportamentais. Por exemplo, um aluno que 
tem uma experiência emocional positiva com um professor, durante um semestre, pode procurar aulas adicionais com o mesmo professor em um semestre subsequente. Da mesma foram, emoções negativas geradas durante um semestre podem afetar negativamente a vontade do aluno de ter aulas adicionais com o mesmo professor.

A imagem e respostas atitudinais são, também, influenciadas pela responsabilidade compartilhada (em torno de $25 \%$ ). Ou seja, uma parte significativa das percepções dos alunos acerca das características do curso de administração que está recebendo (útil, bom, agradável, positivo) é influenciada pela percepção desses mesmos alunos sobre o compartilhamento de responsabilidades de seu aprendizado. Os resultados da pesquisa de Sierra (2009) e deste estudo trazem uma contribuição significativa sobre os efeitos de responsabilidade compartilhada para o conhecimento da aprendizagem do aluno. Sabe-se, portanto, que a responsabilidade compartilhada é um componente crítico do aprendizado do aluno, por levar a respostas atitudinais, comportamentais e emocionais propícias em direção a sua experiência de educação empresarial.

Assim, instituições de ensino superior devem se adequar a essa visão de coproduzir o conhecimento juntamente com o aluno, oportunizando aos professores estruturar seus cursos para promover ambientes colaborativos e cooperativos (PRINCE, 2004).

As limitações deste estudo são primeiramente em relação a sua amostra, pois os dados foram coletados entre estudantes de graduação do curso de Administração de uma instituição de ensino universitário na região de Porto Alegre/RS. Para dar validade externa ao estudo, dados adicionais poderiam ser coletados em diferentes regiões e em outras universidades. Além disso, assim como propôs Sierra (2009), para proporcionar mais conhecimentos sobre as respostas dos alunos para a responsabilidade compartilhada, novas pesquisas poderiam analisar a influência de fatores demográficos, idade, sexo, experiência de vida, etc. 


\section{REFERÊNCIAS}

AUSTER, E. R.; WYLIE, K. K. Creating active learning in the classroom: A systematic approach. Journal of Management Education, v. 30, n. 2, p. 333-353, 2006.

BAILEY, J. J. Students as clients in a professional/client relationship. Journal of Management Education, v. 24, n. 3, p. 353-365, 2000.

BITNER, M. J.; FARANDA, W. T.; HUBBERT, A. R.; ZEITHAML V. A. Customer contributions and roles in service delivery. International Journal of Service Industry Management, v. 8, n. 3, p. 193-205, 1997.

BONWELL, C. C.; EISON, J. A. Active Learning: Creating Excitement in the Classroom. 1991 ASHE-ERIC Higher Education Reports. ERIC Clearinghouse on Higher Education, The George Washington University, One Dupont Circle, Suite 630, Washington, DC 20036$1183,1991$.

BRAMBILLA, F. R.; DAMACENA, C. Cocriação de valor no ensino superior privado: uma análise etnometodológica com alunos de administração de uma universidade do sul do Brasil. Administração: Ensino e Pesquisa, v. 13, n. 3, p. 455-490, 2012.

CARINI, R. M.; KUH, G. D.; KLEIN, S. P. Student engagement and student learning: testing the linkages. Research in Higher Education, v. 47, n. 1, p. 1-32, 2006.

CHUNG, E.; MCLARNEY, C. The classroom as a service encounter: Suggestions for value creation. Journal of Management Education, v. 24, n. 4, p. 484-500, 2000.

CURRAN, J. M.; ROSEN, D. E. Student attitudes toward college courses: An examination of influences and intentions. Journal of Marketing Education, v. 28, n. 2 p. 135-148, 2006.

CROPANZANO, R.; MITCHELL, M. S. Social exchange theory: An interdisciplinary review. Journal of management, v. 31, n. 6, p. 874-900, 2005.

DUKE, C. R. Learning outcomes: Comparing student perceptions of skill level and importance. Journal of Marketing Education, v. 24, n. 3, p. 203-217, 2002.

FORNELL, C.; LARCKER, D. F. Structural equation models with unobservable variables and measurement error: Algebra and statistics. Journal of marketing research, v. 18, n. 3, p. 382-388, 1981.

GIBBS, P. Higher education as a market: a problem or solution?. Studies in Higher Education, v. 26, n. 1, p. 85-94, 2001.

GUSTAFSSON, A.; JOHNSON, M. D.; ROOS, I. The effects of customer satisfaction, relationship commitment dimensions, and triggers on customer retention. Journal of marketing, v. 69, n. 4, p. 210-218, 2005.

HALBESLEBEN, J. R.; BECKER, J. A.; BUCKLEY, M. R. Considering the labor contributions of students: An alternative to the student-as-customer metaphor. Journal of Education for Business, v. 78, n. 5, p. 255-257, 2003. 
HAIR, J. F.; RINGLE, C. M.; SARSTEDT, M. PLS-SEM: indeed a silver bullet. The Journal of Marketing Theory and Practice, v. 19, n. 2, p. 139-152, 2011.

HILL, Y.; LOMAS, L.; MACGREGOR, J. Students' perceptions of quality in higher education. Quality assurance in education, v. 11, n. 1, p. 15-20, 2003.

JOHNSON, D. W., JOHNSON, R. T., SMITH, K. A. Active Learning: Cooperation in the College Classroom (Section 5: $6 \pm$ 9). Edina, MN: Interaction Book. 1991.

KAENDLER, C.; WIEDMANN, M.; RUMMEL, N.; SPADA, H. Teacher competencies for the implementation of collaborative learning in the classroom: a framework and research review. Educational Psychology Review, v. 27, n. 3, p. 505-536, 2015.

KARNS, G. L. Learning style differences in the perceived effectiveness of learning activities. Journal of Marketing Education, v. 28, n. 1, p. 56-63, 2006.

KOTZE, T. G.; PLESSIS, D. P. J. Students as "co-producers" of education: a proposed model of student socialization and participation at tertiary institutions. Quality Assurance in Education, v. 11, n. 4, p. 186-201, 2003.

KU, H.-H.; HSU, K.-H. Effects of inviting customers to share responsibility in the context of impersonal service. Journal of Service Theory and Practice, v. 25, n. 3, p. 267-284, 2015.

LAWLER, E. J.; THYE, S. R.; YOON, J. Emotion and Group Cohesion in Productive Exchange. American Journal of Sociology, v. 106, n. 3, p. 616-657, 2000.

LAWLER, E. J. An Affect Theory of Social Exchange1. American Journal of Sociology, v. 107, n. 2, p. 321-352, 2001.

LYNCH, K. Neo-liberalism and marketisation: The implications for higher education. European Educational Research Journal, v. 5, n. 1, p. 1-17, 2006.

MALHOTRA, N. K. Pesquisa de marketing: uma orientação aplicada. Bookman, 2012.

MOSTAFA, R. B. Engaging Students via Social Media Is It Worth the Effort? Journal of Marketing Education, v. 37, n. 3, p. 144-159, 2015.

NIEMI, H. Active learning-a cultural change needed in teacher education and schools. Teaching and teacher education, v. 18, n. 7, p. 763-780, 2002.

NG, I. C.; FORBES, J. Education as service: the understanding of university experience through the service logic. Journal of Marketing for Higher Education, v. 19, n. 1, p. 38-64, 2009.

PALADINO, A. Creating an interactive and responsive teaching environment to inspire learning. Journal of Marketing Education, v. 30 n. 3, p. 185-188, 2008.

PIKE, G. R.; KUH, G. D.; McCORMICK, A. C. An investigation of the contingent relationships between learning community participation and student engagement. Research in Higher Education, v. 52, n. 3, p. 300-322, 2011.

PRAHALAD, C. K.; RAMASWAMY, V. Co-creation experiences: The next practice in value creation. Journal of interactive marketing, v. 18, n. 3, p. 5-14, 2004. 
PRINCE, M. Does active learning work? A review of the research. Journal of Engineering Education-Washington, v. 93, n.1, p. 223-232, 2004.

SIERRA, J. J. Shared Responsibility and Student Learning Ensuring a Favorable Educational Experience. Journal of Marketing Education, v. 32, n. 1, p. 104-111, 2009.

SIERRA, J. J.; HEISER, R. S.; McQUITTY, S. Exploring determinants and effects of shared responsibility in service exchanges. The Journal of Marketing Theory and Practice, $\mathrm{v}$. 17, n. 2, p. 111-128, 2009.

SIERRA, J. J.; McQUITTY, S. Service providers and customers: social exchange theory and service loyalty. Journal of Services Marketing, v. 19, n. 6, p. 392-400, 2005.

VARGO, S. L.; LUSCH R. F. Evolving to a New Dominant Logic for Marketing. Journal of Marketing, v. 68, n. 1, p. 1-17, 2004.

YOUNG, M. R. The motivational effects of the classroom environment in facilitating self-regulated learning. Journal of Marketing Education, v. 27, n. 1, p. 25-40, 2005.

ZHAO, C.-M.; KUH, G. D. Adding value: Learning communities and student engagement. Research in Higher Education, v. 45, n. 2, p. 115-138, 2004. 


\section{ANEXO A - ITENS DA ESCALA ORIGINAL}

Shared Responsibility (7-point Likert-type scale: $\mathrm{I}=$ strongly dis- agree to $7=$ strongly agree)

I. Because of the important role that I and the professor played in the delivery of my education, I feel as though we worked together as equals to make this learning experience a success.

2. I sensed a certain amount of accountability for the professor and me to make this learning experience end successfully.

3. The professor and I relied on each other to make this learning experience a success.

4. The professor and I were both responsible for the educational outcome of this class.

5. The professor and I were both in control of the educational results in this class.

Emotional Response (7-point Likert-type scale: $\mathrm{I}=$ strongly disagree to 7 = strongly agree)

I. I am happy with the marketing education I received.

2. The marketing education that I received was pleasant.

3. I am satisfied with the marketing education I received.

4. I am content with the marketing education I received.

5. I had an enjoyable marketing education experience.

6. The marketing education I received was gratifying.

Attitudinal Response: 7-point Semantic Differential scale

I. Unfavorable/Favorable

2. Bad $/ \operatorname{good}\left(\mathrm{aTT}_{3}\right)$ Unpleasant/Pleasant

3. Negative/Positive

Intentions: 7-point Semantic Differential scale

I. Would not seek out/Would seek out

2. Not very likely/Very likely

3. Improbable/Probable

4. Would not consider/Would consider

5. Unwilling/Willing

Image: 7-point Semantic Differential scale

I. Not credible/Credible

2. Not prestigious/Prestigious

3. Disreputable/Reputable

4. Irresponsible/Responsible

5. Not trustworthy/Trustworthy

6. Low quality/High quality

7. Unreliable/Reliable appendix 


\section{DADOS DOS AUTORES}

\section{CLÁUDIO DAMACENA` damacena.claudio@gmail.com}

Doutor em Administração pela Universidade de Córdoba (Espanha)

Instituição de vinculação: Pontifícia Universidade Católica do Rio Grande do Sul Porto Alegre/RS - Brasil

Áreas de interesse em pesquisa: Cocriação de valor e inovação em serviços.

*Av. Ipiranga, 6681, Prédio 50, Sala 1102 Partenon Porto Alegre/RS 90619-900

\section{THAYS MARTINS DO NASCIMENTO thaysdn@gmail.com} Bacharel em Administração com ênfase em Marketing pela PUCRS

Instituição de vinculação: Pontifícia Universidade Católica do Rio Grande do Sul Porto Alegre/RS - Brasil

Áreas de interesse em pesquisa: Comportamento do consumidor. 